Copyright @ 2009 Institute of Electrical and electronics Engineers, Inc.

All Rights reserved.

Personal use of this material, including one hard copy reproduction, is permitted.

Permission to reprint, republish and/or distribute this material in whole or in part for any other purposes must be obtained from the IEEE.

For information on obtaining permission, send an e-mail message to stds-igr@ieee.org.

By choosing to view this document, you agree to all provisions of the copyright laws protecting it.

Individual documents posted on this site may carry slightly different copyright restrictions.

For specific document information, check the copyright notice at the beginning of each document. 


\title{
Time-delay Effect on Equivalent Control Based Single-Input Sliding Mode Control Systems
}

\author{
Xinghuo Yu, Qinglong Han, Xiangjun Li, Changhong Wang
}

\begin{abstract}
This paper studies the time-delay effect on the equivalent control based sliding mode control. Conditions to guarantee the boundedness of the control system steady states under the time delayed equivalent control based sliding mode control are obtained. Maximum upper bound of the delay time to guarantee boundedness is estimated. Simulations are conducted to verify the theoretical results.
\end{abstract}

\section{INTRODUCTION}

Sliding mode control (SMC) systems have been extensively studied and successfully applied to solving many industrial problems [1]. The essence of SMC lies in the effort that in a vicinity of a prescribed switching manifold, the velocity vectors of the controlled state trajectories always point toward the switching manifold. An ideal sliding mode exists only when the system state satisfies the dynamic equation that governs the sliding mode for all time. This requires an infinite switching in general. It is known that, if the SMC is implemented in networked control environment, it is inevitable that there will be communication delay in control signals, which may deteriorate significantly the elegant invariance property enjoyed by the continuous-time SMC. The question is to what extent such influence is detrimental? Little research has been done except for some related results in discretization of SMC systems ([2], [3]).

In this paper, we will study the time-delay effect on the most popular SMC - the equivalent control based SMC systems. Conditions to guarantee the boundedness of the control system under the time delayed equivalent control based sliding mode control are obtained. Maximum upper bound of the delay time to guarantee stability is estimated. Digital simulations are conducted to verify the theoretical results.

\section{THE SINGLE-INPUT EQUIVALENT CONTROL BASED SMC SYSTEM}

Consider the following controllable single-input linear SMC system with a switching manifold $\sigma$ :

$$
\begin{aligned}
\dot{x} & =A x+b u, \\
\sigma(x) & =c^{\top} x,
\end{aligned}
$$

$\mathrm{X}$. Yu is with School of Electrical and Computer Engineering, RMIT University, Melbourne, Australia. Email: x.yu@ rmit.edu.au

Q. Han is Faculty of Business and Informatics, Central Queensland University, Rockhampton, Australia. Email: q.han@cqu.edu.au

$\mathrm{X}$. Li is with Department of Control Science and Engineering, Harbin Institute of Technology, China. Email: xiangjun.li@rmit.edu.au

C. Wang is with Department of Control Science and Engineering, Harbin Institute of Technology, China. Email: chwang@hit.edu.cn where $x \in R^{n}, u, g \in R^{1}, A$ is an $n \times n$ matrix, and $b$ and $c$ are $n$-dimensional vectors, respectively. The switching manifold $\sigma$ is predefined to represent a desired asymptotically stable dynamics. Its corresponding equivalent control based SMC is

$$
\begin{aligned}
u & =u_{e q}+u_{\sigma}, \\
u_{e q} & =-\left(c^{\top} b\right)^{-1} c^{\top} A x, \\
u_{\sigma} & =-\alpha\left(c^{\top} b\right)^{-1} \operatorname{sgn} \sigma(x),
\end{aligned}
$$

with $\alpha>0$ being a constant control gain, sgn the sign function, and $c^{\top} b \neq 0$. Without loss of generality, it is assumed that $c^{\top} b=1$.

Note [1] that the equivalent control $u_{e q}$ is derived by solving $\dot{\sigma}=0$ subject to (1). It can be easily verified that for the Lyapunov function $V=\frac{1}{2} \sigma^{2}$, the time derivative of $V$ along the dynamics (1) with (3), (4) and (5) yields $\dot{V}=$ $-\alpha|\sigma|=-\alpha V^{1 / 2}$, which indicates the finite time attainability and global stability of $\sigma=0$.

The control law (3) is by far the most popular SMC structure. For simplicity, and without loss of generality, we assume that the systems are in the controllable canonical form, that is,

$$
\begin{gathered}
A=\left(\begin{array}{ccccc}
0 & 1 & 0 & \ldots & 0 \\
0 & 0 & 1 & \ldots & 0 \\
\vdots & \vdots & \vdots & \vdots & \vdots \\
0 & 0 & 0 & \ldots & 1 \\
-a_{1} & -a_{2} & -a_{3} & \ldots & -a_{n}
\end{array}\right) \\
b=\left(\begin{array}{lllll}
0 & 0 & \ldots & 0 & 1
\end{array}\right)^{\top} \\
c=\left(\begin{array}{lllll}
c_{1} & c_{2} & \ldots & c_{n-1} & 1
\end{array}\right)^{\top}
\end{gathered}
$$

Under these assumptions, substituting the control (3) with (5) and (II) leads to

$$
\dot{x}=A_{e q} x-\alpha b \operatorname{sgn} \sigma(x)
$$

where

$$
A_{e q}=\left(\begin{array}{ccccc}
0 & 1 & 0 & \ldots & 0 \\
0 & 0 & 1 & \ldots & 0 \\
\vdots & \vdots & \vdots & \vdots & \vdots \\
0 & 0 & 0 & \ldots & 1 \\
0 & -c_{1} & -c_{2} & \ldots & -c_{n-1}
\end{array}\right) \text {, }
$$

Note that $\sigma=0$ is an $(n-1)$-dimensional dynamics whose characteristic equation is

$$
\lambda^{n-1}+c_{n-1} \lambda^{n-2}+\cdots+c_{2} \lambda+c_{1}=0
$$


Normally, the coefficients $c_{1}, c_{2}, \ldots, c_{n-1}$ are chosen so that solutions of (9) have negative real parts, i.e. the polynomial (9) is Hurwitz. The characteristic polynomial of $A_{e q}$ is

$$
\lambda\left(\lambda^{n-1}+c_{n-1} \lambda^{n-2}+\cdots+c_{2} \lambda+c_{1}\right)=0
$$

where the root $\lambda=0$ corresponds to the 'fast' motion leading to the sliding mode and the rest of roots correspond to the 'slow' motion when the system is in the sliding mode.

Note also the matrix $A_{e q}$ can be written as

$$
\begin{aligned}
A_{e q} & =\left(A-b c^{T} A\right) \\
& =\left(\begin{array}{ccccc}
0 & 1 & 0 & \cdots & 0 \\
0 & 0 & 1 & \cdots & 0 \\
\vdots & \vdots & \ddots & \ddots & \vdots \\
0 & 0 & \cdots & 0 & 1 \\
0 & -c_{1} & \cdots & -c_{n-2} & -c_{n-1}
\end{array}\right) \\
& =\left(\begin{array}{cc}
0 & e_{1}^{T} \\
0 & B_{c}
\end{array}\right), \\
e_{1}=(1,0, \cdots, 0)^{T} \in R^{n-1}, \text { and } & \\
B_{c} & =\left(\begin{array}{cccc}
0 & 1 & \cdots & 0 \\
\vdots & \ddots & \ddots & \vdots \\
0 & \cdots & 0 & 1 \\
-c_{1} & \cdots & -c_{n-2} & -c_{n-1}
\end{array}\right)
\end{aligned}
$$

The SMC system is designed in such a way that in ideal situation, the sliding manifold $\sigma=0$ will be reached in finite time. The subsystem $y=\left(x_{2}, x_{3}, \ldots x_{n}\right)^{T}$ is asymptotically stable because the eigenvalues of $B_{c}$ are zeros of the characteristic equation (9) which is Hurwitz.

\section{THE TIME-DELAYED SINGLE-INPUT EQUIVALENT CONTROL BASED SMC SYSTEM}

Under the time delay influence in the control channel, the SMC system (1) becomes:

$$
\dot{x}(t)=A x(t)+b u(t-\tau)
$$

Substituting the time delayed control $u(t-\tau)$ in (3), (12) becomes

$$
\begin{aligned}
\dot{x}(t)= & A_{e q} x(t)+b c^{\top} A(x(t)-x(t-\tau)) \\
& -\alpha b \operatorname{sgn} \sigma(x(t-\tau))
\end{aligned}
$$

It can be easily seen that

$$
\dot{\sigma}=-c^{\top} A(x(t)-x(t-\tau))-\alpha \operatorname{sgn} \sigma(x(t-\tau))
$$

In order to ensure that the time-delayed single-input equivalent control based SMC system maintains the essential properties of the sliding mode and the boundedness of the overall system steady states, we need to ensure that the matrix

$$
A_{e q} x(t)-b c^{\top} A(x(t)-x(t-\tau))
$$

has all its eigenvalues on the left hand side of the complex plane (including the imaginary axis). For the convenience of study, we decompose the system in the following.

Since matrices

$$
A_{e q}=\left(\begin{array}{cc}
0 & e_{1}^{T} \\
0 & B_{c}
\end{array}\right)
$$

and because

$$
\begin{aligned}
b c^{\top} A & =\left(\begin{array}{cccc}
0 & 0 & \cdots & 0 \\
0 & 0 & \cdots & 0 \\
\vdots & \ddots & \ddots & \vdots \\
-a_{1} & c_{1}-a_{2} & \cdots & c_{n-1}-a_{n}
\end{array}\right) \\
& =\left(\begin{array}{cc}
0 & 0 \\
e_{2} & B_{\tau}
\end{array}\right)
\end{aligned}
$$

where $z=\left(x_{2}, \cdots, x_{n}\right)^{T}, e_{2}=\left(0, \cdots, 0,-a_{1}\right)^{T} \in R^{n-1}$, and

$$
B_{\tau}=\left(\begin{array}{ccc}
0 & \cdots & 0 \\
\vdots & \ddots & \vdots \\
0 & \cdots & 0 \\
c_{1}-a_{2} & \cdots & c_{n-1}-a_{n}
\end{array}\right) \in R^{(n-1) \times(n-1)}
$$

the time delayed equation (13) can be rewritten as

$$
\begin{aligned}
\dot{x}_{1}(t)= & x_{2}(t) \\
\dot{z}(t)= & B_{c} z(t)+B_{\tau}(z(t)-z(t-\tau)) \\
& -a_{1} \bar{b}\left(x_{1}(t)-x_{1}(t-\tau)\right) \\
& -\alpha \bar{b} \operatorname{sgn} \sigma(x(t-\tau))
\end{aligned}
$$

where $\bar{b}=(0, \cdots, 0,1)^{T} \in R^{n-1}$.

It can be easily seen that if $\tau=0$, then the system becomes

$$
\begin{aligned}
\dot{x}_{1}(t) & =x_{2}(t) \\
\dot{z}(t) & =B_{c} z(t)-\alpha \bar{b} \operatorname{sgn} \sigma(x(t))
\end{aligned}
$$

which is the same as (7), an asymptotically stable motion. The sliding mode $\sigma=0$ will be reached in finite time.

The linear part of the subsystem $z$ is $\dot{z}(t)=B_{c} z(t)$. Since $B_{c}$ is asymptotically stable, all its eigenvalues have negative real parts. With the same expectation, we hope the subsystem

$$
\dot{z}(t)=B_{c} z(t)+B_{\tau}(z(t)-z(t-\tau))
$$

have all the roots of the characteristic polynomial are on the left hand side of the complex plane which is equivalent to its asymptotically stability. On the other hand, since $B_{c}$ is designed to be a Hurwitz matrix, so there will be a $\tau_{\max }$ such that when $\tau<\tau_{\max }$, subsystem (17) will be asymptotically stable. In the following, we state and establish a theorem for such stability.

Theorem 1: The system (17) is asymptotically stable if there exist real $(n-1) \times(n-1)$ matrices $P>0, Q>0, R>0$, $W>0$, and $S_{11}=S_{11}^{T}, S_{12}, S_{22}=S_{22}^{T}$ satisfying $S=S^{T} \geq 0$ such that

$$
\begin{aligned}
& \left(\begin{array}{cccc}
(1,1) & W+S_{12} & -P B_{\tau}+R & 0.5 \tau\left(B_{c}+B_{\tau}\right)^{T} W \\
* & (2,2) & -S_{12} & 0 \\
* & * & (3,3) & -0.5 \tau B_{\tau}^{T} W \\
* & * & * & -W \\
* & * & * & *
\end{array}\right. \\
& \left.\begin{array}{c}
\tau\left(B_{c}+B_{\tau}\right)^{T} R \\
0 \\
-\tau B_{\tau}^{T} R \\
0 \\
-R
\end{array}\right)<0
\end{aligned}
$$


where

$$
\begin{aligned}
& (1,1) \triangleq\left(B_{c}+B_{\tau}\right)^{T} P+P\left(B_{c}+B_{\tau}\right)+Q+S_{11}-W-R \\
& (2,2) \triangleq S_{22}-S_{11}-W \\
& (3,3) \triangleq-S_{22}-Q-R
\end{aligned}
$$

In order to prove Theorem 1, we need the following lemma. Lemma 1: [6] For any constant matrix $W \in \mathbb{R}^{n \times n}, W=$ $W^{T}>0$, scalar $\tau>0$, and vector valued function $\dot{z}:[-\tau, 0] \rightarrow$ $\mathbb{R}^{n}$ such that the following integration is well defined, then

$$
\begin{aligned}
& -\tau \int_{-\tau}^{0} \dot{z}^{T}(t+\xi) W \dot{z}(t+\xi) d \xi \\
& \leq\left(\begin{array}{lll}
z^{T}(t) & z^{T}(t-\tau)
\end{array}\right)\left(\begin{array}{cc}
-W & W \\
W & -W
\end{array}\right)\left(\begin{array}{c}
z(t) \\
z(t-\tau)
\end{array}\right) .
\end{aligned}
$$

Proof: Choosing the following Lyapunov-Krasovskii functional

$$
\begin{aligned}
V\left(t, z_{t}\right) & =z^{T}(t) P z(t)+\int_{t-\tau}^{t} z^{T}(\xi) Q z(\xi) d \xi \\
& +\int_{t-\tau}^{t}(\tau-t+\xi) \dot{z}^{T}(\xi)(\tau R) \dot{z}(\xi) d \xi \\
& +\int_{t-0.5 \tau}^{t} y^{T}(\xi) S y(\xi) d \xi \\
& +\int_{t-0.5 \tau}^{t}(0.5 \tau-t+\xi) \dot{z}^{T}(\xi)(0.5 \tau W) \dot{z}(\xi) d \xi
\end{aligned}
$$

Taking the derivative of $V\left(t, z_{t}\right)$ with respect to $t$ along the trajectory of (17) yields

$$
\begin{aligned}
\dot{V}\left(t, z_{t}\right)= & z^{T}(t)\left[\left(B_{c}+B_{\tau}\right)^{T} P+P\left(B_{c}+B_{\tau}\right)+Q\right] z(t) \\
& -2 z^{T}(t) P B_{\tau} z(t-\tau)-z^{T}(t-\tau) Q z(t-\tau) \\
& +y^{T}(t) S y(t)-y^{T}(t-0.5 \tau) S y(t-0.5 \tau) \\
& +\dot{z}^{T}(t)\left(\tau^{2} R\right) \dot{z}(t)+\dot{z}^{T}(t)\left(0.25 \tau^{2} W\right) \dot{z}(t) \\
& -\int_{t-\tau}^{t} \dot{z}^{T}(\xi)(\tau R) \dot{z}(\xi) d \xi \\
& -\int_{t-0.5 \tau}^{t} \dot{z}^{T}(\xi)(0.5 \tau W) \dot{z}(\xi) d \xi .
\end{aligned}
$$

Use (17) to obtain

$$
\dot{z}^{T}(t)\left(\tau^{2} R\right) \dot{z}(t)=\zeta^{T}(t) \Xi_{B}^{T}\left(\tau^{2} R\right) \Xi_{B} \zeta(t),
$$

and

$$
\dot{z}^{T}(t)\left(0.25 \tau^{2} W\right) \dot{z}(t)=\zeta^{T}(t) \Xi_{B}^{T}\left(0.25 \tau^{2} W\right) \Xi_{B} \zeta(t),
$$

where

$$
\begin{aligned}
& \Xi_{B}=\left(\left(B_{c}+B_{\tau}\right) 0-B_{\tau}\right) \\
& \zeta^{T}(t)=\left(\begin{array}{lll}
z^{T}(t) & z^{T}(t-0.5 \tau) & z^{T}(t-\tau)
\end{array}\right) .
\end{aligned}
$$

By Lemma 1, one has

$$
\begin{aligned}
& -\int_{t-\tau}^{t} \dot{z}^{T}(\xi)(\tau R) \dot{z}(\xi) d \xi \\
& \leq\left(\begin{array}{c}
z(t) \\
z(t-\tau)
\end{array}\right)^{T}\left(\begin{array}{cc}
-R & R \\
R & -R
\end{array}\right)\left(\begin{array}{c}
z(t) \\
z(t-\tau)
\end{array}\right),
\end{aligned}
$$

and

$$
\begin{aligned}
& -\int_{t-0.5 \tau}^{t} \dot{z}^{T}(\xi)(0.5 \tau W) \dot{z}(\xi) d \xi \\
& \leq y^{T}(t)\left(\begin{array}{cc}
-W & W \\
W & -W
\end{array}\right) y(t)
\end{aligned}
$$

Then from (20) to (24), we have

$$
\dot{V}\left(t, z_{t}\right) \leq \zeta^{T}(t) \Xi \zeta(t),
$$

where

$$
\Xi=\left(\begin{array}{ccc}
\Xi_{11} & \Xi_{12} & \Xi_{13} \\
* & \Xi_{22} & \Xi_{23} \\
* & * & \Xi_{33}
\end{array}\right),
$$

with

$$
\begin{aligned}
\Xi_{11}= & \left(B_{c}+B_{\tau}\right)^{T} P+P\left(B_{c}+B_{\tau}\right)+Q+S_{11}-W-R \\
& +\left(B_{c}+B_{\tau}\right)^{T}\left(\tau^{2} R+0.25 \tau^{2} W\right)\left(B_{c}+B_{\tau}\right), \\
\Xi_{12}= & W+S_{12}, \\
\Xi_{13}= & -P B_{\tau}+R-\left(B_{c}+B_{\tau}\right)^{T}\left(\tau^{2} R+0.25 \tau^{2} W\right) B_{\tau}, \\
\Xi_{22}= & S_{22}-S_{11}-W, \\
\Xi_{23}= & -S_{12}, \\
\Xi_{33}= & -S_{22}-Q-R+B_{\tau}^{T}\left(\tau^{2} R+0.25 \tau^{2} W\right) B_{\tau} .
\end{aligned}
$$

Notice that in light of Schur complement, (19) is equivalent to $\Xi<0$. Therefore, there exists a scalar $\lambda>0$ such that $\dot{V}\left(t, z_{t}\right) \leq-\lambda z^{T}(t) z(t)<0$ for $z(t) \neq 0$, which means that the system (17) is asymptotically stable. This completes the proof.

While Theorem 1 gives conditions for the stability of system (17), when switching element is involved, the asymptotical stability cannot be realized. The time delay effect will result in certain oscillatory motions within a bounded region. We present the following theorem for the globally convergent boundedness of the system states.

Theorem 2: If conditions in Theorem 1 hold, and

$$
\left|a_{1}\left(x_{1}(t)-x_{1}(t-\tau)\right)\right|<\infty,
$$

then $y$ in system (15) will be convergent and eventually bounded. Furthermore, if

$$
\int_{0}^{\infty} x_{2}(t) d t<\infty
$$

then the overall system will be convergent and eventually bounded.

Proof: The subsystem $z$ in system (15) can be viewed as a linear system with a bounded input $v(t)=a_{1}\left(x_{1}(t)-x_{1}(t-\right.$ $\tau))+\alpha \operatorname{sgn} \sigma(x(t-\tau))$ with input matrix $\bar{b}$. Since under the conditions of theorem 1 , the unforced system is exponentially stable, therefore the system will be input-to-state stable [7]. If $x_{2}$, which is the first item of $z$, also satisfies $\int_{0}^{\infty} x_{2}(t) d t<\infty$, then we will have

$$
x_{1}(t)=x_{1}(0)+\int_{0}^{\infty} x_{2}(t) d t<\infty .
$$

The eventual boundedness of $z$ and $x_{1}$ implies the eventual boundedness of $x$.

Note that the stability of overall system is closely related to the stability of subsystem (17). Furthermore, Theorems 2 and 1 give us a way to estimate the delay constant enabling the stability (boundedness). In the following, we will use it to estimate the maximum time delay for ensuring stability (boundedness) in a simulation. 


\section{SIMULATION STUDIES}

Consider a third order single-input system which is

$$
\begin{aligned}
\dot{x}_{1}= & x_{2} \\
\dot{x}= & B_{c} \underline{x}+B_{\tau}(\underline{x}(t)-\underline{x}(t-\tau)) \\
& -a_{1} \bar{b}\left(x_{1}(t)-x_{1}(t-\tau)\right)-\alpha \bar{b} \operatorname{sgn} \sigma(x(t-\tau))
\end{aligned}
$$

where

$$
B_{c}=\left(\begin{array}{cc}
0 & 1 \\
-c_{1} & -c_{2}
\end{array}\right), \quad B_{\tau}=\left(\begin{array}{cc}
0 & 0 \\
c_{1}-a_{2} & c_{2}-a_{3}
\end{array}\right),
$$

and $\bar{b}=\left(\begin{array}{ll}0 & 1\end{array}\right)^{\mathrm{T}}$.

We consider two scenario:

1) $a_{1}=1, a_{2}=2, a_{3}=1, c_{1}=1, c_{2}=2$ and $\alpha=1$. That is the original system and the sliding mode are all asymptotically stable. using Theorem 1 to calculate the maximum time delay gives $\tau_{\max }=1.4142$. Set the initial condition as $x(0)=\left(\begin{array}{lll}0.5 & 0.5 & 0.5\end{array}\right)^{\mathrm{T}}$ and $\tau=0.1$. The state trajectory of subsystem (17) is shown in Figure $1(a)$. The state trajectory of system (27) and $\sigma(t)$ are shown in Figures $1(b)$ and $(c)$ respectively.

2) $a_{1}=1, a_{2}=-2, a_{3}=1, c_{1}=1, c_{2}=2$ and $\alpha=1$. That is the original system is unstable but the the sliding mode is asymptotically stable. use Theorem 1 to calculate $\tau_{\max }=0.64$. Set the initial condition as $x(0)=\left(\begin{array}{lll}0.5 & 0.5 & 0.5\end{array}\right)^{\mathrm{T}}$ and $\tau=0.1$. The state trajectory of subsystem (17) is shown in Figure $2(a)$. The state trajectory of system (27) and $\sigma(t)$ are shown in Figure $2(b)$ and $(c)$ respectively.

Note that the sliding variable exhibits periodic oscillations around the zero point, satisfying the condition $\int_{0}^{\infty} x_{2}(t) d t<\infty$ in Theorem 2. Through these two examples, it can be seen that the asymptotical stability of the subsystem and the boundness of the time-delayed SMC system are guaranteed.

\section{CONClusion}

In this paper, time-delayed effect on equivalent control based SMC system has been studied. A LMI condition has been obtained to guarantee the stability of the 'slow' subsystem in the SMC system, and conditions to guarantee the stability of the entire system have also been given. An estimation method of the maximum delay time has been given as well.

\section{ACKNOWLEDGMENT}

The first author is grateful for a grant from the Australian Research Council.

\section{REFERENCES}

[1] V. I. Utkin, Sliding Modes in Optimization and Control, Springer Verlag, New York, 1993.

[2] X. Yu and G. Chen, "Discretization behaviors of equivalent control based sliding-mode control systems," IEEE Transactions on Automatic Control, vol. 48, no. 9, pp. 1641-1646, 2003.

[3] Z. Galias and X. Yu, "Euler's discretization of single input sliding mode control systems," IEEE Transactions on Automatic Control, vol. 52, pp. 1726-1730, 2007.

[4] C. Edwards and S. Spurgeon, Sliding Mode Control: Theory and Applications, Taylor and Francis, London, 1998.



(a)

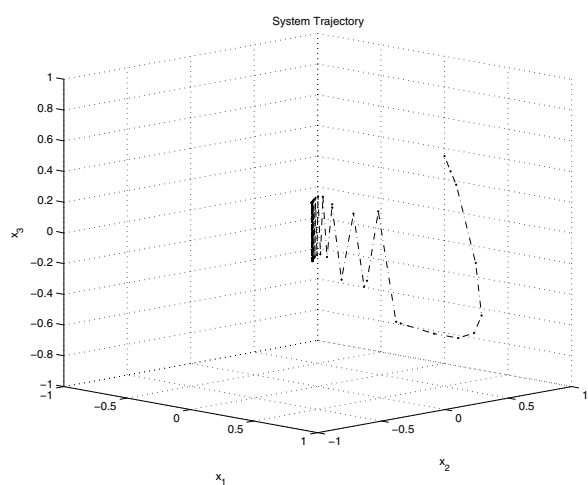

(b)

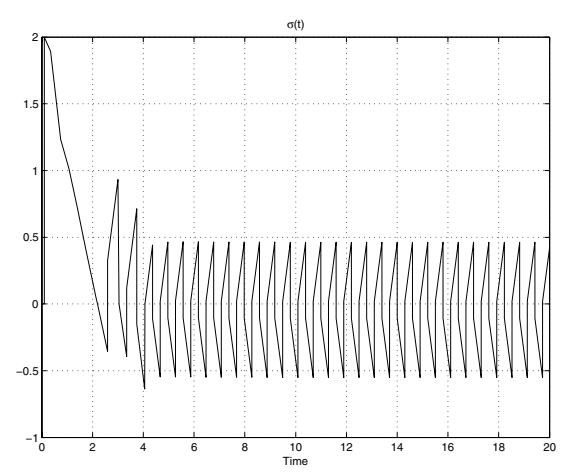

(c)

Fig. 1. (a) State trajectory of subsystem (17); (b) State trajectory of system (27); (c) $\sigma(t)$.

[5] A. Sabanovic, L.M. Fridman and S. Spurgeon, Variable Structure Systems: From Principles to Implementation, IEE, London, 2004.

[6] Q.-L. Han, "Absolute stability of time-delay systems with sectorbounded nonlinearity," Automatica, vol. 41, no. 12, pp. 2171-2176.

[7] Y. Nima, D. Michel and Y. Nader, "Relation between exponential stability and input-to-state stability of time-delay systems," in Proc. Of the 2007 ACC, New York, America, 2007. 




(a)



(b)



(c)

Fig. 2. (a) State trajectory of subsystem (17); (b) State trajectory of system (27); (c) $\sigma(t)$. 